\title{
SECOND ORDER ESTIMATES IN INTERPOLATION THEORY AND APPLICATIONS
}

\author{
MARIO MILMAN AND TOMAS SCHONBEK
}

(Communicated by William J. Davis)

\begin{abstract}
We provide a new approach to the commutator theorems of Rochberg and Weiss [10], Jawerth, Rochberg, and Weiss [6], Cwikel, Jawerth, and Milman [4]. In particular our methods apply to non-linear operators of a type commonly encountered in classical harmonic analysis (maximal operators, square functions). As an application we provide an extension of a commutator inequality of Chanillo [3] for maximal operators of fractional integrals.
\end{abstract}

\section{INTRODUCTION}

Given a compatible pair of Banach spaces $\bar{A}$ the classical constructions of interpolation theory provide methods to obtain parametrized families of spaces, with the property that if $T$ is a bounded linear operator on the starting pair, then $T$ also will be bounded on the interpolation spaces. Associated with these constructions are certain operators, $\Omega$, generally unbounded and nonlinear, which can be obtained by differentiation with respect to certain parameters used in the specific method. For example, in the case of the $L^{p}$ spaces, such operators are given by $\Omega f=f \log \left(|f| /\|f\|_{p}\right)$ or by $\Omega f=f \log r_{f}(x)$, where $r_{f}(x)=\mid\{y:|f(y)|>|f(x)|$ or $|f(y)|=|f(x)|, y \leq x\} \mid$. It is shown in [10] and [5] that if $T: \bar{A} \rightarrow \bar{A}$ is a bounded linear operator, $F$ denotes the real or complex method of interpolation, and $\Omega=\Omega_{F}$ denotes the corresponding associated operator, then the commutator $[T, \Omega]=T \Omega-\Omega T$ is bounded in the corresponding spaces $F(\bar{A})$ as well as on the domain of definition of $\Omega$.

We refer to [5] for a detailed survey including an application to logarithmic Sobolev inequalities. Among the applications given in [10] we mention the proof that the commutators of singular integrals of Calderón-Zygmund type and multiplication by BMO functions are bounded in $L^{p}$. In [4] it is shown that one can obtain an explicit characterization of the domain spaces associated with the real interpolation methods.

In this paper we provide a new, elementary, and possibly simpler approach to these results. Moreover, our methods allow us to deal with nonlinear operators

Received by the editors June 19, 1989 and, in revised form, January 9, 1990.

1980 Mathematics Subject Classification (1985 Revision). Primary 46M35; Secondary 42B25.

Key words and phrases. Interpolation theory, non-linear operators, commutators. 
and thus provide a solution to a problem proposed in [5]. As an example we extend a result of Chanillo [3] on commutation between fractional integrals and multiplication by BMO functions to maximal fractional integral operators. We also indicate how to handle some of the more general operators $\Omega$ considered by Kalton in [7].

The plan of the paper is as follows. In $\S 2$ we provide some background and definitions and state the commutator theorems. These results are then proved, in a slightly more general form, in $\S 3$. In $\S 4$ we prove a nonlinear version of the commutator theorem and provide some applications. Finally, $\S 5$ contains some further results that can be derived with our methods.

\section{OPTIMAL DECOMPOSITIONS AND QUASILOGARITHMIC OPERATORS}

In this paper we focus mainly on the $K$ method of interpolation. By the observations of [6], [4] and [5], our results can be seen to imply the corresponding commutator results for the $J$ and $E$ methods of interpolation. Therefore, we shall only review briefly the definitions for the $K$ method and refer the reader to the papers quoted above for more details on the corresponding theory for the other real methods (cf. also (3.11) below). For background information on interpolation theory we refer to [1].

Let $\bar{A}=\left(A_{0}, A_{1}\right)$ be a compatible pair of Banach spaces, which from now on we shall simply refer to as a "pair." Recall that the $K$ functional is the norm of the space $\Sigma_{t}(\bar{A})=A_{0}+t A_{1}$ for each $t>0$, i.e.,

$$
K(t, f, \bar{A})=K(t, f)=\inf \left\{\left\|f_{0}\right\|_{A_{0}}+t\left\|f_{1}\right\|_{A_{1}}: f=f_{0}+f_{1}, f_{i} \in A_{i}, i=0,1\right\} .
$$

The interpolation spaces $\bar{A}_{\theta, q, K}=\bar{A}_{\theta, q}$ are defined for $0<\theta<1,0<q \leq$ $\infty$, by

$$
\bar{A}_{\theta, q}=\left\{f \in \Sigma(\bar{A}):\|f\|_{\theta, q}=\phi_{\theta, q}(K(t, f))<\infty\right\},
$$

where the function norm $\phi_{\theta, q}$ is defined on measurable functions on $(0, \infty)$ by

$$
\phi_{\theta, q}(h)=\left\{\int_{0}^{\infty}\left[t^{-\theta}|h(t)|\right]^{q} d t / t\right\}^{1 / q} .
$$

The purpose of the so-called local theory of interpolation is to study certain operators associated with the computation of the $K$ functional. Thus, we make the following definition (cf. [6]): for each $t>0$, we say that $f=f_{0}(t)+f_{1}(t)$ is an almost optimal decomposition if

$$
\left\|f_{0}(t)\right\|_{A_{0}}+t\left\|f_{1}(t)\right\|_{A_{1}} \leq c K(t, f),
$$

where $c$ is a constant whose value is fixed beforehand, say $c=2$. Given an almost optimal decomposition we write $D_{K}(t) f=f_{0}(t)$. Associated with any such choice we have an operator $\Omega_{\bar{A}, K}=\Omega$, defined by

$$
\Omega f=\int_{0}^{1} D_{K}(t) f d t / t-\int_{1}^{\infty}\left(I-D_{K}(t)\right) f d t / t .
$$


We observe that, although $\Omega$ is well defined on $\bar{A}_{\theta, q}, 0<\theta<1,0<q \leq \infty$, it is not bounded on these spaces. In [4] these operators $\Omega$ are called "quasilogarithmic" operators. For a given $\bar{A}_{\theta, q}$, we let $\operatorname{Dom}_{\theta, q}(\Omega)=\operatorname{Dom}(\Omega)=\{f \in$ $\left.\bar{A}_{\theta, q}: \Omega f \in \bar{A}_{\theta, q}\right\}$ and $\|f\|_{\operatorname{Dom}_{\theta, q}(\Omega)}=\|f\|_{\operatorname{Dom}(\Omega)}=\|f\|_{\theta, q}+\|\Omega f\|_{\theta, q}$.

The basic abstract results concerning these constructions are the following two theorems.

(2.1) Theorem. (cf. [6]). (i) Let $\bar{A}, \bar{B}$, be Banach pairs, and let $T$ be a bounded linear operator, $T: \bar{A} \rightarrow \bar{B}$. Then $[T, \Omega]=T \Omega_{\bar{A}}-\Omega_{\bar{B}} T$ is a bounded operator from $\bar{A}_{\theta, q}$ to $\bar{B}_{\theta, q}, 0<\theta<1,0<q \leq \infty$.

(ii) $T$ is bounded from $\operatorname{Dom}_{\theta, q}\left(\Omega_{\bar{A}}\right)$ to $\operatorname{Dom}_{\theta, q}\left(\Omega_{\bar{B}}\right)$.

(2.2) Theorem. (cf. [4]). For a pair $\bar{A}$, and $\theta, q$, as in (2.1), let $\bar{A}_{\log , \theta, q}=$ $\left\{f \in \bar{A}_{\theta, q}:\|f\|_{\log , \theta, q}=\phi_{\theta, q}(K(t, f)(1+|\log t|))<\infty\right\}$. Then $\operatorname{Dom}_{\theta, q}\left(\Omega_{\bar{A}}\right)=$ $\bar{A}_{\log , \theta, q}$.

Observe that (2.2) implies, in particular, (2.1)(ii).

\section{Proof AND extension of (2.1) AND (2.2)}

The original proof of (2.1) was based on the equivalence between the $K$ and $J$ methods of interpolation (i.e., the so-called "fundamental lemma"). One of the problems with this approach is that it is not clear how to extend the result to nonlinear operators. Concerning (2.2), the original arguments reduced the proof, through the use of local retracts, to the proof of the corresponding result for weighted $L^{p}$ spaces. We provide here elementary proofs based on properties of optimal decompositions and Hardy's inequality.

For the purpose of further extensions, we consider more general quasilogarithmic operators. Let $\theta \in(0,1), q \in(0, \infty)$ and assume $w$ is a positive function on $(0, \infty)$ which is a "good weight" for the Hardy type inequalities, i.e., such that

$$
\begin{gathered}
\phi_{\theta, q}\left(\int_{0}^{t} f(s) w(s) d s\right) \leq c \phi_{\theta, q}(f), \\
\phi_{\theta, q}\left(t \int_{t}^{\infty} f(s) w(s) d s / s\right) \leq c \phi_{\theta, q}(f)
\end{gathered}
$$

hold for some $c=c(\theta, q)$ and for all functions $f$. We then define, for a given pair $\bar{A}$,

$$
\Omega_{u} f=\Omega_{\bar{A}, u} f=\int_{0}^{1} D_{K}(t) f w(t) d t-\int_{1}^{\infty}\left(I-D_{K}(t)\right) f w(t) d t,
$$

where $D_{K}(\cdot)$ is an almost optimal decomposition. We set $\operatorname{Dom}\left(\Omega_{w}\right)=\{f \in$ $\left.\bar{A}_{\theta, q}: \Omega_{w} f \in \bar{A}_{\theta, q}\right\}$. Let $\nu=\nu_{w}$ be defined by $\nu(t)=\int_{1}^{t} w(s) d s$. In particular, if $w(t)=1 / t$, then $\Omega_{w}=\Omega, \nu(t)=\log t$ and (3.1), (3.2) hold. 
(3.3) Theorem. Suppose that $w$ satisfies (3.1) and (3.2) for a given $(\theta, q) \in$ $(0,1) \times(0, \infty]$. Then

$$
D\left(\Omega_{w}\right)=\left\{f \in \bar{A}_{\theta, q}:\|f\|_{\nu, \theta, q}=\phi_{\theta, q}\{(1+|\nu(\cdot)|) K(\cdot, f)\}<\infty\right\} .
$$

Proof. Let $f \in \bar{A}_{\theta, q}$. A simple rearrangement of the integrals defining $\Omega_{w}$ gives

$$
\Omega_{w} f=\int_{0}^{t} D_{K}(s) f w(s) d s-\int_{t}^{\infty}\left(I-D_{K}(s)\right) f w(s) d s-\nu(t) f .
$$

Therefore, using the triangle inequality,

$K\left(t, \Omega_{w} f+\nu(t) f\right) \leq \int_{0}^{t} K\left(t, D_{K}(s) f\right) w(s) d s+\int_{t}^{\infty} K\left(t,\left(I-D_{K}(s)\right) f\right) w(s) d s$.

It follows readily from the definitions that the following estimates hold (cf. [2]):

$$
\begin{gathered}
K\left(t, D_{K}(s) f\right) \leq\left\|D_{K}(s) f\right\|_{A_{0}} \leq c K(s, f), \\
K\left(t,\left(I-D_{K}(s)\right) f\right) \leq t\left\|\left(I-D_{K}(s)\right) f\right\|_{A_{1}} \leq c(t / s) K(s, f) .
\end{gathered}
$$

Thus, we get

$$
K\left(t, \Omega_{w} f+\nu(t) f\right) \leq c \int_{0}^{t} K(s, f) w(s) d s+c t \int_{t}^{\infty} K(s, f) w(s) \frac{d s}{s} .
$$

Applying $\phi_{\theta, q}$ to (3.4) and using (3.1), (3.2), we obtain

$$
\phi_{\theta, q}\left(K\left(t, \Omega_{w} f+\nu(t) f\right)\right) \leq c \phi_{\theta, q}(K(t, f)) .
$$

Using once again the fact that $K(t, \cdot)$ is a norm and (3.5), we have

$$
\phi_{\theta, q}\left(K\left(t, \Omega_{w} f\right)-|\nu(t)| K(t, f)\right) \leq c \phi_{\theta, q}(K(t, f)) .
$$

It follows readily from (3.6) that $\phi_{\theta, q}\left(K\left(\cdot, \Omega_{w} f\right)\right)<\infty$ if and only if

$$
\phi_{\theta, q}(|\nu(\cdot)| K(\cdot, f))<\infty,
$$

proving the theorem.

We now extend (2.1) as follows.

(3.7) Theorem. Let $\theta, q, w, \nu$ be as above. If $\bar{A}$ and $\bar{B}$ are pairs and $T: \bar{A} \rightarrow \bar{B}$ is linear and bounded, then, for some constant $c$ and for all $f \in A_{\theta, q}$,

$$
\left\|\left[\Omega_{w}, T\right]\right\|_{\theta, q}=\left\|\left(\Omega_{\bar{B}, w} T-T \Omega_{\bar{A}, w}\right) f\right\|_{\theta, q} \leq c\|f\|_{\theta, q} .
$$

Proof. Let $f \in \bar{A}_{\theta, q}$. For $t>0$,

$$
\begin{aligned}
& \phi_{\theta, q}\left(K\left(t, \Omega_{\bar{B}, w} T f-T \Omega_{\bar{A}, w} f\right)\right) \\
& \quad \leq \phi_{\theta, q}\left(K\left(t, \Omega_{\bar{B}, w} T f+\nu(t) T f\right)\right)+\phi_{\theta, q}\left(K\left(t, \nu(t) T f+T \Omega_{\bar{A}, w} f\right)\right) .
\end{aligned}
$$

The first term can now be estimated using (3.5) and the fact that $T$ is bounded on $\bar{A}_{\theta, q}$. The second term can be estimated using the linearity of $T$, the fact that it preserves the $K$ functional, and (3.5). The result follows. 
(3.9) Remark. Notice that the assumption $T: \bar{A} \rightarrow \bar{B}$ was used only to estimate the second term in the right hand side of (3.8); the first term was estimated using only the fact that $T: \bar{A}_{\theta, q} \rightarrow \bar{B}_{\theta, q}$. This observation can be used to obtain a slightly more general version of (2.1), cf. (5.2) below.

(3.10) Remark. The weights $w$ for which (3.1) or (3.2) holds have been characterized by Muckenhoupt [8] so that it is not difficult to come up with some non-trivial choices satisfying (3.1) and (3.2) for some $q$ and all $\theta \in(0,1)$. Observe, oriented toward the results of Kalton, that one such choice is

$$
w(t)=\frac{\alpha}{t|\log t|^{1-\alpha}}
$$

which is valid for $0<\theta<1, q \geq 1,1 / q<\alpha \leq 1$. In this case, $|\nu(t)|=$ $|\log t|^{\alpha}$, and the corresponding operator $\Omega_{w}$ for the pair $\left(L^{1}, L^{\infty}\right)$ can be defined by

$$
\Omega_{w} f=-f \log r_{f}\left|\log r_{f}\right|^{\alpha-1} .
$$

(3.11) Remark. There are other quasilogarithmic operators generated by other methods of interpolation. For the $J$ method it is shown in [4] that $\Omega_{J}=$ $-\Omega_{K}$, and therefore all the results here and in the next section also hold for these operators. Similar ideas can be used to deal with operators $\Omega=\Omega_{E}$ obtained through the use of the $E$ method of interpolation. For this method the corresponding $\Omega$ 's are defined by

$$
\Omega f=\int_{1}^{\infty} D_{E}(t) f d t / t-\int_{0}^{1}\left(I-D_{E}(t)\right) f d t / t .
$$

\section{COMMUTATOR THEOREMS FOR NONLINEAR OPERATORS ACTING ON LATTICES}

Let $\bar{A}$ be a pair of Banach spaces and $D_{K}(\cdot)$ an almost optimal decomposition. We define the operators $\Omega_{0}, \Omega_{1}$ by

$$
\Omega_{0} f=\Omega_{\bar{A}, 0} f=\int_{0}^{1} D_{K}(t) f \frac{d t}{t}, \Omega_{1} f=\Omega_{\bar{A}, 1} f=\int_{1}^{\infty}\left(I-D_{K}(t)\right) f \frac{d t}{t} .
$$

Then $\Omega=\Omega_{0}-\Omega_{1}$. The same type of arguments which proved (3.4) prove (4.1)

$$
K\left(t, \Omega_{0} f-\left(\log _{+} \frac{1}{t}\right) f, \bar{A}\right) \leq c\left\{\int_{0}^{t} K(s, f \bar{A}) \frac{d s}{s}+t \int_{t}^{\infty} K(s, f, \bar{A}) \frac{d s}{s^{2}}\right\}
$$

$$
K\left(t, \Omega_{1} f-\left(\log _{+} t\right) f, \bar{A}\right) \leq c\left\{\int_{0}^{t} K(s, f, \bar{A}) \frac{d s}{s}+t \int_{t}^{\infty} K(s, f, \bar{A}) \frac{d s}{s^{2}}\right\} .
$$

Now assume $\bar{A}$ and $\bar{B}$ are pairs of Banach lattices; and, let $T: \bar{A} \rightarrow \bar{B}$ be a (non-linear) operator such that $T f \geq 0, T(\alpha f)=|\alpha| T f$, for $f \in$ $\Sigma(\bar{A})$, scalar $\alpha$, and satisfying the pointwise estimate $|T f(x)-T g(x)| \leq$ $|T(f-g)(x)|$. For example, these conditions are satisfied by operators $T$ ob- 
tained as the pointwise supremum of sublinear operators, in particular, most classical maximal functions, sharp functions, area functions, $g$ functions, etc.

(4.3) Theorem. Let $T: \bar{A} \rightarrow \bar{B}$ be as above. For $0<\theta<1,0<q \leq \infty$, there is a $c=c(\theta, q)$ such that

$$
\left\|\left[\Omega_{i}, T\right]\right\|_{\theta, q}=\left\|\Omega_{\bar{B}, i} T f-T \Omega_{\bar{A}, i} f\right\|_{\theta, q} \leq c\|f\|_{\theta, q},
$$

for $i=0,1, f \in \Sigma(\bar{A})$.

Proof. Let $t>0$, and let $\nu(t)=\log _{+}(1 / t)$ if $i=0, \nu(t)=\log _{+} t$ if $i=1$. Then

$$
\begin{aligned}
\left|\Omega_{\bar{B}, i} T f-T \Omega_{\bar{A}, i} f\right| & \leq\left|\Omega_{\bar{B}, i} T f-\nu(t) T f\right|+\left|T(\nu(t) f)-T \Omega_{\bar{A}, i} f\right| \\
& \leq\left|\Omega_{\bar{B}, i} T f-\nu(t) T f\right|+\left|T\left(\nu(t) f-\Omega_{\bar{A}, i} f\right)\right| .
\end{aligned}
$$

Applying $K(t, \cdot, \bar{B})$ to the last inequality and using the readily verified fact that $K(t, T g, \bar{B}) \leq c K(t, g, \bar{A})$, we get, in view of (4.1), (4.2),

$$
K\left(t,\left[\Omega_{i}, T \mid f, \bar{B}\right) \leq c\left\{\int_{0}^{t} K(s, f, \bar{A}) \frac{d s}{s}+t \int_{t}^{\infty} K(s, f, \bar{A}) \frac{d s}{s^{2}}\right\} .\right.
$$

The result follows applying $\phi_{\theta, q}$.

Restricting to the case $\bar{A}=\bar{B}$, it is now easy to see that if one $\Omega_{i}$ is bounded in $\bar{A}_{\theta, q}$, then $[\Omega, T]$ is bounded for all nonlinear operators $T: \bar{A} \rightarrow \bar{A}$ satisfying the conditions above. For example, if there is a $c \geq 0$ such that $\left\|\Omega_{1} f\right\|_{\theta, q} \leq c\|f\|_{\theta, q}$ for $f \in \bar{A}_{\theta, q}$, then

$$
\begin{aligned}
|[\Omega, T] f| & \leq\left|\left(\Omega_{0}-\Omega_{1}\right) T f-\Omega_{0} T f\right|+\left|\Omega_{0} T f-T\left(\Omega_{0}-\Omega_{1}\right) f\right| \\
& \leq\left|\Omega_{1} T f\right|+\left|\left[\Omega_{0}, T\right] f\right|+\left|T \Omega_{0} f-T\left(\Omega_{0}-\Omega_{1}\right) f\right| \\
& \leq\left|\Omega_{1} T f\right|+\left|\left[\Omega_{0}, T\right] f\right|+\left|T \Omega_{1} f\right| .
\end{aligned}
$$

Since $\Omega_{1}$ and $T$ are bounded in $\bar{A}_{\theta, q}$, we conclude that $[\Omega, T]$ is bounded in $\bar{A}_{\theta, q}$ by (4.3). An important example, in which $\Omega_{1}$ is bounded in $\bar{A}_{\theta, q}$ for all $(\theta, q) \in(0,1) \times(0, \infty]$, is the case $A_{1} \subset A_{0}$ (i.e., the pair is "ordered"). This can be seen as follows: in the situation at hand we can choose almost optimal decompositions in such a way that $\left(I-D_{K}(t)\right) f=0$, for $t>1$, thus the difference between the $\Omega$ constructed using this decomposition and any other $\Omega$ is equal to $\Omega_{1}$ and is bounded by the commutator theorem (2.1)(i) applied to the identity. Consequently, from (4.3) we get

(4.4) Theorem. Let $\bar{A}$ be an ordered lattice pair and let $T: \bar{A} \rightarrow \bar{A}$ be a nonlinear operator as above. For every $(\theta, q) \in(0,1) \times(0, \infty]$ there exists $c=c(\theta, q)$ such that

$$
\|[\Omega, T] f\|_{\theta, q}=\|\Omega T f-T \Omega f\|_{\theta, q} \leq c\|f\|_{\theta, q} .
$$

(4.5) Remark. For an ordered pair it is readily seen that $\Omega_{1}$ is actually bounded from $\Sigma(\bar{A})=A_{0}$ to $\Delta(\bar{A})=A_{1}$. This follows from the direct estimate $\left\|\Omega_{1} f\right\|_{A_{1}} \leq c\|f\|_{A_{0}} \int_{1}^{\infty} d s / s^{2}=c\|f\|_{A_{0}}$. However, let us observe that both $\Omega_{i}$ 's cannot be bounded in $\bar{A}_{\theta, q}$ at the same time unless the pair is trivial, i.e., $A_{0}=A_{1}$ (cf. [5]). 
As an application, let $b \in \mathrm{BMO}, b \geq 0$, and $w=e^{b}$. Then $L^{p}(w d x) \subset$ $L^{p}\left(w^{-1} d x\right)$ and $\Omega f=-(2 / p) b f$. Recall that if the BMO norm of $b$ is small enough, then $w$ and $w^{-1}$ belong to the Muckenhoupt class $A_{p}$ and, consequently, the Hardy-Littlewood maximal operator $M$ is bounded on $L^{p}\left(w^{ \pm 1} d x\right)$. We can then apply (4.4) to the pair $\bar{A}=\left(L^{p}\left(w^{-1} d x\right), L^{p}(w d x)\right)$ with $q=p$ and $\theta=1 / 2$, using a homogeneity argument to remove the restriction on the BMO norm of $b$. Since $\bar{A}_{1 / 2, p}=L^{p}(d x)$, we get

Theorem. If $b \geq 0$ is in $\mathrm{BMO}$, then

$$
\|M(b f)-b M(f)\|_{L^{p}} \leq c\|b\|_{\mathrm{BMO}}\|f\|_{L^{p}}, \quad 1<p<\infty .
$$

Similar results hold for the sharp maximal function, etc.

Let $M_{\gamma}$ be the fractional maximal operator defined by

$$
M_{\gamma} f(x)=\sup _{x \in Q}|Q|^{-1+\gamma / n} \int_{Q}|f(x)| d x .
$$

Using an argument similar to the one above, together with results concerning the boundedness of $M_{\gamma}$ on weighted $L^{p}$ spaces (due to Muckenhoupt and Wheeden [9]), we find

(4.7) Theorem. Let $0<\gamma<n, 1<p<n / \gamma, 1 / q=1 / p-\gamma / n$, and $0 \leq$ $b \in \mathrm{BMO}$. Let $T_{\gamma}$ denote the usual fractional integral operator or the maximal fractional operator $M_{\gamma}$ (as defined above). Then

$$
\left\|T_{\gamma}(b f)-b T_{\gamma}(f)\right\|_{L^{a}} \leq c\|b\|_{\mathrm{BMO}}\|f\|_{L^{p}}, \quad 1<p<\infty .
$$

The commutator theorem for fractional integral operators is due to Chanillo [3], and a proof using complex interpolation commutators was given in [10]. For the maximal operators $M_{\gamma}$ the result seems to be new.

It follows that (4.4) makes the local theory of interpolation available to deal with the familiar nonlinear operators of classical analysis. We leave as an open question whether it is possible to remove the restriction that the pair $\bar{A}$ be ordered in (4.3). For a different approach towards answering this question see [5].

\section{FURTHER EXTENSIONS AND REMARKS}

A. We consider now conditions under which we can weaken the assumptions of the commutator theorem in different directions. We assume only that $T$ is a bounded linear operator (there are of course some nonlinear versions of this which we shall not consider here) on one interpolation space $\bar{A}_{\theta_{0}, q_{0}}$, for some fixed values of $\theta_{0}$ and $q_{0}$, and in addition that $T$ satisfies the following condition: there exists a constant $c>0$ such that if $h:(0, \infty) \rightarrow \Sigma(\bar{A})$ satisfies $\phi_{\theta_{0}, q_{0}}(K(., h()))<.\infty$, then

$$
\phi_{\theta_{0}, q_{0}}(K(., T(h(.)))) \leq c \phi_{\theta_{0}, q_{0}}(K(., h(.))) .
$$

Clearly, (5.1) holds if $K(t, T f) \leq c K(t, f)$ for $f \in \Sigma(\bar{A}), t>0$. We have 
(5.2) Theorem. Assume $T$ satisfies (5.1), and let $\Omega=\Omega_{w}$. Then there exists an absolute constant $c=c(\theta, q)>0$ such that

$$
\|\Omega T-T \Omega\|_{\theta_{0}, q_{0}} \leq c\|f\|_{\theta_{0}, q_{0}} .
$$

Proof. Proceed as in the proof of (3.7) but, instead of using the boundedness of $T$ on $\bar{A}$ to estimate the second term of the right hand side of (3.8), use (5.1).

B. Higher order commutators. The process of taking commutators can, in principle, be iterated. Thus, one is led to consider $[[T, \Omega], \Omega]$, etc. In the theory developed in previous papers it was not possible to do this unless $\Omega$ could be chosen to be linear (i.e., the quasilinearizable case). We present a somewhat more general result here.

(5.3) Lemma. Let $H$ be a (possibly) nonlinear operator, acting on a pair $\bar{A}$, and such that

(i) $\|H f\|_{\theta, q} \leq c\|f\|_{\theta, q}$,

(ii) $\phi_{\theta, q}(K(t, H f \nu(t)+H \Omega f)) \leq c\|f\|_{\theta, q}$.

Then

Proof. Add and subtract $H f \nu(t)$.

$$
\|[H, \Omega] f\|_{\theta, q} \leq c\|f\|_{\theta, q} .
$$

(5.4) Lemma. If $\Omega$ satisfies the property

$$
\phi_{\theta, q}(K(t, \Omega f \nu(t)+\Omega \Omega f)) \leq c\|f\|_{\theta, q},
$$

then $H=[\Omega, T]$, where $T$ is a bounded operator on $\bar{A}$, satisfies the conditions of (5.3).

Proof. The usual computation.

In particular (5.4) holds for linear $\Omega$ 's.

\section{REFERENCES}

1. J. Bergh and J. Lofstrom, Interpolation spaces : an introduction, Springer-Verlag, Berlin, Heidelberg, and New York, 1976.

2. J. H. Bramble and R. Scott, Simultaneous approximation in scales of Banach spaces, Math. Comp. 32 (1978), 947-954.

3. S. Chanillo, A note on commutators, Indiana Math. J. 31 (1982), 7-17.

4. M. Cwikel, B. Jawerth, and M. Milman, The domain spaces of quasilogarithmic operators, Trans. Amer. Math. Soc. (to appear).

5. M. Cwikel, B. Jawerth, M. Milman, and R. Rochberg, Differential estimates and commutators in interpolation theory, Analysis at Urbana II, London Math. Soc. Lecture Notes, vol. 138, Cambridge Univ. Press, 1989, pp. 170-220.

6. B. Jawerth, R. Rochberg, and G. Weiss, Commutator and other second order estimates in real interpolation theory, Ark. Mat. 24 (1986), 191-219.

7. N. Kalton, Nonlinear commutators in interpolation theory, Mem. Amer. Math. Soc., no. 73, Amer. Math. Soc., Providence, RI, 1988. 
8. B. Muckenhoupt, Hardy's inequality with weights, Studia Math. 44 (1972), 31-38.

9. B. Muckenhoupt and R. Wheeden, Weighted norm inequalities for fractional integrals, Trans. Amer. Math. Soc. 192 (1974), 261-274.

10. R. Rochberg and G. Weiss, Derivatives of analytic families of Banach spaces, Ann. Math. 118 (1983), 315-347.

Department of Mathematics, florida Atlantic University, Boca Raton, Florida 33431 